\title{
Fluid Balance Monitoring Accuracy in Intensive Care Units
}

\author{
Hayam I. Asfour \\ Department of Critical Care and Emergency Nursing, Faculty of Nursing, Alexandria University, Alexandria, \\ Egypt
}

\begin{abstract}
Introduction: Fluid balance monitoring is a part of the scope of nurses' practice. The incorrect calculation of fluid balance means that every patient management decision was influenced by inaccurate fluid balance information. Therefore, nurses working in ICUs are responsible and accountable for the accurate recording and calculation of fluid balance when caring for critically ill patients.

The aim of the work to examine the accuracy of fluid balance monitoring in critical care units and determine the factors affecting fluid balance monitoring in critical care units.

The study design was a descriptive research design.

Setting, This study was carried out in two general intensive care units (ICUs) at a selected Hospital in Alexandria- Egypt.

Subjects, fluid balance related records for 100 critical care patient and 60 critical care nurses was drawn from the previously mentioned ICUs.

Data collection instrument; the tool of this study was "Fluid balance monitoring accuracy in ICUs" This tool combined two parts: "Fluid balance monitoring audit" and "Fluid balance monitoring questionnaire".

Results; $65 \%$ of fluid balance recorded in patients' folders were accurate and 35\% were inaccurate. The level of nurses' knowledge regarding fluid balance monitoring in ICUs was moderately adequate (61.67\%) and $71 \%$ of nurses had favorable perception regarding fluid balance monitoring in ICUs Inaccuracy of the measuring equipment / continuous intravenous infusions, time management (100\%), workload (91.67\%) and lack of skills/ training (33.33\%) were factors affecting fluid balance monitoring as reported by nurses.

Conclusion: Two thirds of the recorded fluid balance was accurate and the inaccurate fluid balance recorded was fluctuating between positive and negative balance. Nurses reported that inaccuracy of the measuring equipment / continuous intravenous infusions, time management, workload, and lack of skills/ training are factors affecting fluid balance monitoring in ICU.

Recommendations: For accuracy of fluid balance monitoring in ICU, increase awareness and in-service education should be conducted to improve nurses' knowledge. Novice nurses should be trained adequately regarding assessment and monitoring of fluid balance.
\end{abstract}

Keywords: Fluid balance - Monitoring-Critical care nurses

Fluid Balance Monitoring Accuracy in Intensive Care Units

Fluid balance monitoring is an essential part of the process of care of critically ill patients. Fluid Balance is the balancing of intake and output of fluid to permit metabolic processes to function correctly. Maintaining fluid balance plays an important role in the managing critically ill patients. The accurate monitoring of fluid balance activities is a vital part of patients' baseline information, which guides medical and nursing interventions to achieve physiological stability. Inaccurate monitoring of fluid balance especially in critically ill patients can deteriorate patients' conditions ${ }^{(1)}$. Therefore, fluid balance should be monitored and recorded accurately for patients in intensive care units (ICUs).

A 24-h period of a day fluid balance is among important measures recorded in patients' daily observation sheet, which are nursing interventions, vital signs, medical procedures. The balance is the difference between the intake and output volumes. Monitoring patients' fluid balance is of great importance in managing and understanding patients' clinical status ${ }^{(1-3)}$. Studies were focused on the fluid accumulation consequences in critically ill patients, such as hypertension, tissue and peripheral edema, respiratory failure, and increased cardiac demand $^{(3-7)}$.

Several studies conducted in ICU have suggested that fluid overload has been linked with adverse outcomes ${ }^{(3-8)}$. It was found that at least one day of negative fluid balance is a predictor of survival in patients with septic shock. In addition, studies found that a positive mean daily fluid balance was a significant predictor of mortality in ICU. However, a more positive fluid balance (in both resuscitation and cumulatively over 4 days) is associated with an increased risk of mortality ${ }^{(3-8)}$. Payen et al. (2008) found that mean ICU fluid balance was independently linked with a high risk of ICU mortality ${ }^{(7)}$. 
Fluid balance monitoring is a part of the scope of nurses' practice. Therefore, nurses working in ICUs are responsible and accountable for the accurate recording and calculation of fluid balance when caring for critically ill patients. Managing patients' fluid balance is as equally important as carrying out any other patient care activity for the critically ill ${ }^{(8)}$.

The effective management of critically ill patients requires accurate assessment of their fluid balance status. This assessment includes appropriate monitoring of fluid intake and output, as well as the accurate calculation and correct recording of this data ${ }^{(2,3)}$. In an informal pilot study of fluid balance records of critically ill patients, seven out of ten of these fluid balance calculations were incorrect. In this pilot study, no fluid balance calculation was available for one patient of them at all. More than a quarter of fluid balance records has a deviation between the researchers' calculation and the recorded calculation and this deviation was more than $500 \mathrm{ml}^{(9)}$.

The incorrect calculation of fluid balance means that every patient management decision was influenced by inaccurate fluid balance information. Therefore, fluid balance monitoring requires close attention to provide comprehensive data, upon which patient management decisions can be based ${ }^{(7,9,10)}$. In addition, studies showed that fluid balance calculation is not treated as a priority in nursing management of critically ill patients $^{(10-15)}$.

Similarly, Perren et al. (2011) ${ }^{(4)}$ expressed their concern about fluid balance accuracy in critically ill patients. Additionally, the researchers found that there is a higher chance of the calculated fluid balance being incorrect ${ }^{(4)}$. This finding supports many studies findings that a careful and accurate approach to fluid balance monitoring does not appreciate high priority in managing critically ill patients ${ }^{(10-12)}$.

Inaccurate fluid balance monitoring and recording can result in complications with respect to on-going patients' management. Inaccurate fluid balance data may result in inappropriate administration of diuretic therapy, resulting in fluid imbalances that affect the patients' hemodynamic stability. It is essential that critical care nurses implement appropriate correct recording, accurate calculation and fluid balance monitoring, to provide safe patient care ${ }^{(5,6,11)}$. Therefore, this study aimed to examine the accuracy of fluid balance monitoring in critical care units and determine the factors affecting fluid balance monitoring in critical care units.

\section{Study questions were:}

- Is monitoring fluid balance in ICU accurate?

- What are the level of critical care nurses' knowledge regarding fluid balance monitoring in the ICU?

- What are the critical care nurses' perception regarding fluid balance monitoring in the ICU?

- What are the factors affecting fluid balance monitoring in the ICU?

Materials:

\section{Materials and method}

Research design: A descriptive design was used in this study.

Setting: This study was carried out in two general intensive care units (ICUs) at a selected Hospital in Alexandria- Egypt. Patients were admitted to the ICUs directly from emergency department or transferred from other hospital departments. Patients had a variety of disorders.

Subjects: the study combined two parts; "Fluid balance monitoring audit" (part 1) and "Fluid balance monitoring questionnaire" (part 2).

Part 1: "Fluid balance monitoring audit" The subjects for the audit were all critical care fluid balance related records for 100 patients with the following inclusion criteria; nursing records of admissions to critical care from January to April 2012, and nursing records of the first 48 hours of a patient's stay in the ICU, when available. Patients on dialysis were excluded, as they need special monitoring. Subjects were selected and divided specifically among the ICUs under the guidance of the statistician.

Part 2: "Fluid balance monitoring questionnaire". A convenient sample of 60 critical care nurses was drawn from the previously mentioned ICUs. Nurses less than a year ICU experience and intern nurses were included in the study. Nurses have the ultimate responsibility and accountability for the patients allocated to their care in the ICU.

Data collection instrument; the tool of this study was "Fluid balance monitoring accuracy in ICUs " which was used to collect data regarding accuracy of monitoring fluid balance in ICUs and it was developed by the researcher after reviewing the relevant literatures ${ }^{(1,3,9,10-15)}$. This tool combined two parts:

Part 1 "Fluid balance monitoring audit" This part of the tool was used to assess the current practices implemented in fluid balance monitoring. The audit included all critical care patient fluid balance related 
records, which were daily chart, medication prescription form, and non-medication prescription form, fluid balance related forms, other doctor order forms and the laboratory result.

The audit examined the main parts of fluid balance monitoring:

- Hemodynamic parameters such as vital signs such as pulse, blood pressure and respiratory rate, monitoring of patients' electrolytes values.

- Capillary refill time, skin elasticity (turgor).

- Recording of input prescription and administration in the form of intravenous infusions, enteral feeding, total parenteral nutrition, and blood products.

- Recording of output in the form of urine, vomiting, diarrhea and drainage

- Medications also were monitored especially diuretics.

Intake, output and fluid balance were monitored from observations and patients' folders.

The audit also includes an observation part in which the researcher observe nurses' current fluid balance practices to identify the actual fluid balance. The observation contains the basic items that nurses should perform such as check the amount, type of intake (intravenous fluids, feeding, blood products, others) against prescription, check the amount, type of output (urine, vomiting, drainage and others), adjust the intravenous infusions flow rate accurately, document the time started/ended for intravenous infusions, document the prescribed intake on chart, monitor the exact amount of intake/output.

The researcher calculated the actual fluid balance from observation/prescription and compared it with the recorded in patients file. If both the researcher calculation and the recorded fluid balance matched, the calculated fluid balance was accepted as accurate and if it is not matched fluid balance recorded was considered not accurate.

Part 2: "Fluid balance monitoring questionnaire". This part of the tool was used to identify the knowledge, perception of critical care nurses regarding fluid balance monitoring and factors affecting fluid balance monitoring in the ICU.

It comprised three sections:

Section 2-a: nurses' knowledge regarding fluid balance monitoring. It was used to identify critical care nurses' knowledge regarding fluid balance monitoring. This section includes sixteen multiple-choice questions for eight items with only one possible answer to each question. These questions were developed from the available current literature. The items includes questions about the amount of adequate urine output, the amount of fluid intake an adult patient requires per day (on average), which vital signs are usually part of assessing patient's fluid balance assessment, clinical signs are most likely to occur in a patient who is hypovolemic/hypervolmic, nurses' action for patients who has hypovolemia/ hypervolemia, fluids or drugs infused continuously, blood products included in the fluid balance and factors affecting urine output.

Each question had two levels of responses as the correct answer for any item was given score of one and incorrect answer was given a score of zero. The possible score range was 0 to 16 . A high score indicates good knowledge. Adequate knowledge $\geq 75 \%$ of total score and $<75 \%-60 \%$ moderately adequate knowledge while $<60 \%$ of the total score was considered poor knowledge.

Section 2-b: nurses' perception regarding fluid balance monitoring. It comprised ten statements requiring nurses' responses to determine critical care nurses' perception on fluid balance monitoring. Nurses were asked to indicate their level of agreement with these statements with reference to their ICUs.

The items included in this section were; there are many other patient care activities that are more important for nurse than recording the intake and output, there are too many people who fill in one patient's fluid balance chart, nurses may be responsible for more than one patient, so it is difficult to supervise all the fluid balance activities, nurses are satisfied with the design of the fluid balance chart sheet as it is straightforward to complete, the space to write the fluid numbers on the chart is adequate, the 24-hour fluid balance is correctly calculated all the time, fluid balance assessment is important to guide nursing care in critically ill patients, inaccurate fluid balance calculation can be a risk for the critically ill patient, fluid balance information is recorded in too many different places on critical care observation \& patient records, and the nurses is the only person responsible for a correct fluid balance calculation.

Scoring system: The tool has a 5-point Likert scale ranging from (strongly disagree=1), 2 (disagree $=2$ ), (uncertain $=3$ ), (agree $=4$ ) to (strongly agree $=5$ ). The possible score range was 1 to 50. Favorable perception was $\geq 60 \%$ of total score and $<60 \%$ was considered unfavorable. 
Section 2-c was developed to collect data regarding factors affecting fluid balance monitoring in ICUs. It includes items regarding workload, time management, inaccuracy of the measuring equipment, lack of knowledge / skills / training and communication with other health-care workers.

Scoring system: If the factor is affecting fluid balance monitoring, the response is yes and no is the response when the factor is not affecting fluid balance monitoring. In addition, this part include an open question regarding nurses' recommendations for accurate fluid balance monitoring. Critical care nurses' characteristics namely; age, gender, qualification, years of experience, current position, level of education, patients to nurse ratio were collected.

\section{Tool validity and reliability}

Validity. The study tools were developed after reviewing the related literatures. To determine content and face validity, ten critical care-nursing experts evaluated the tool. Six experts were academic experts in critical care nursing education and critical care medicine. Four experts were drawn from the hospital and ICUs: clinical practice, quality assurance, and education. Each of the experts was an active participant in their particular environment and together they offered a complete assessment of the content and face validity of the instruments. The following recommendations were made based on experts' opinion: the questions that were not appropriate to ask nurses were taken out and some questions needed clarification and modifications to be easier for nurses to understand.

Reliability. To ensure consistency, the researcher and two research assistants collected all the data themselves. This means that the same persons used the same tool in the two ICUs. Tool reliability was tested using internal consistency methods (Alpha Cronbach test). The reliability coefficients were $(r=0.905)$ which indicates an accepted reliability of the tool.

Pilot study. After having the ethical approval and permission to access the hospital, a pilot study was conducted on a sample of $10 \%$ of the subjects. The pilot study was conducted in the same ICUs from the beginning of January to the end of February 2012. All the obtained data in the pilot study were excluded from the main study data. The necessary modifications of the tool were done according to pilot results to reach the finalized form.

\section{Data collection process}

This study started from the beginning of January to the end of June 2012. The researcher trained tworesearch assistances to aid in data collection. Inter-observer consistency exceeding $90 \%$ was confirmed before the study. The researcher and research assistances began with introducing themselves to nurses in the ICUs and providing clear explanation about the nature, aim and purpose of the study. Each nurse was informed that sharing in this study is voluntary. Oral approval of nurses to share in this study was achieved. The study was achieved through two phases.

The first phase (audit/observation phase): the accuracy of fluid balance monitoring was determined utilizing part I of the tool (fluid balance monitoring audit). The researcher used the audit to examine the main parts of fluid balance monitoring; hemodynamic parameters, electrolytes values, intake, output and fluid balance from patients' folders. The researchers also observed nurses' current fluid balance practices to identify the actual fluid balance. The researcher compared the actual fluid balance with the recorded fluid balance in patients' folders. This phase took two months from beginning of March to the end of April 2012.

The second phase (knowledge/perception phase): this phase began after the audit/observation phase to avoid contamination of data. The purpose of this phase was to collect data regarding the knowledge and perception of critical care nurses regarding fluid balance monitoring in ICUs. Nurses were informed (oral and written) about the aim of the study. Critical care nurses were interviewed on an individual basis during the break time in the morning and/or evening shift to identify their knowledge and perception regarding fluid balance monitoring using part 2 of the tool. In addition, nurses were asked to mention if there are any factors affecting fluid balance monitoring and their recommendations to improve fluid balance monitoring. Time spent for each interview ranged from 25 to 30 minutes using the previously stated tool in their break time. This phase took two months from beginning of May to the end of June 2012.

\section{Administrative design and ethical considerations}

The study conducted over a period of 6 months from the beginning of January to the end of June 2012. An official letter clarifying the purpose and setting of the study was obtained from the Nursing Faculty - University of Alexandria and from the hospital administrative authority to conduct the study. Each nurse was informed 
about the significant and aim of the study and then an informed written consent was obtained from each nurse before participating in the study.

Respect for persons was applied through the consideration that nurses participated voluntarily in the study and could withdraw at any time, as there was no obligation to participate. Anonymity and confidentiality were applied as the following; a reference number on the audit was used to allow the researcher to track the study documentation during data analysis, no patients' records were identified in the document audit and no copies were made of the documents, and patient names or numbers were also not recorded on the audit form. Only patient records meeting the inclusion criteria of the study were accessed.

\section{Statistical Analysis}

The raw data were coded and transformed into coding sheets. The results were checked. Then, the data were entered into SPSS system files (SPSS package version 17) using personal computer. Output drafts were checked against the revised coded data for typing and spelling mistakes. Finally, analysis and interpretation of data were conducted. The following statistical measures were used: Descriptive statistics including frequency, distribution, mean, and standard deviation were used to describe different characteristics. Kolmogorov - Smirnov test was used to examine the normality of data distribution. Univariate analyses including: t-test was used to test the significance of results of quantitative variables. Moreover, Chi-Square test and Fisher's exact test were used to test the significance of results of qualitative variables. A significant $p$-value was considered when $p$ less than .05 .

\section{Results}

Figures (1-a \& 1-b) show the accuracy of fluid balance monitoring in critical care units. It was observed that $65 \%$ of fluid balance recorded in patients' folders were accurate and the rest of them (35\%) were inaccurate (figure 1-a). Of the inaccurate fluid balance, seventeen records were positive with the mean and standard deviation $467.65 \pm 161.99 \mathrm{ml}$ and 18 records were negative with the mean and standard deviation $337.78 \pm 151.23 \mathrm{ml}$ (figure $1-b)$.

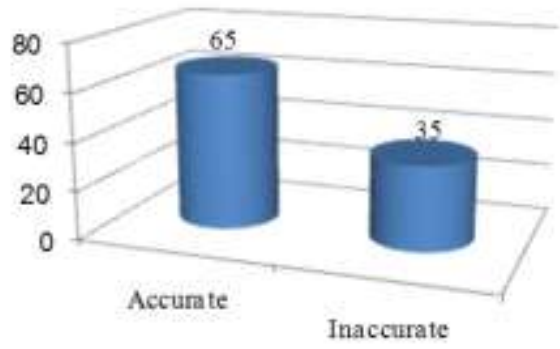

Figure (1-a) the accuracy of fluid balance monitoring in critical care units.

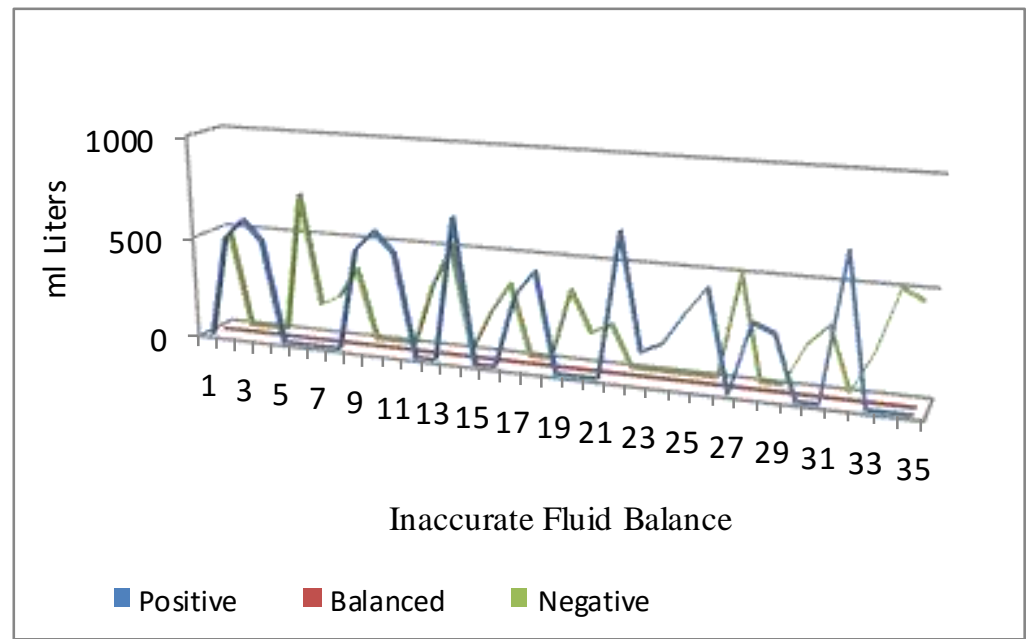

Figure 1-b Distribution of inaccurate fluid balance records 
Table (I): Critical care nurses' knowledge regarding fluid balance monitoring in ICUs. From this table, it was noted that the level of nurses' knowledge regarding fluid balance monitoring in ICUs was moderately adequate $(61.67 \%)$. Nurses have knowledge in some items regarding fluid balance monitoring, which are fluids or drugs infused continuously (91.67\%), clinical signs associated with hypovolemia/ hypervolemia (66.67\%) and which vital signs are a part of fluid balance assessment patient $(60 \%)$. However, nurses knowledge regarding other items of fluid balance monitoring is not enough especially; average amount of urine output (58.33\%), factors affecting urine output (58.33\%), nurses' action for patients who has hypovolemia/ hypervolemia $(41.67 \%)$ and the required amount of fluid intake an adult patient per day (41.67\%).

Table (I) Critical care nurses' knowledge regarding fluid balance monitoring in ICUs

\begin{tabular}{|l|l|l|l|l|}
\hline Knowledge items & \multicolumn{3}{l|}{ Responses (n=60) } \\
\cline { 2 - 6 } & \multicolumn{2}{l|}{ Correct } & \multicolumn{2}{l|}{ Incorrect } \\
\cline { 2 - 6 } & $\mathbf{N}$ & $\mathbf{\%}$ & $\mathbf{N}$ & $\mathbf{\%}$ \\
\hline Average amount of urine output & 25 & 41.67 & 35 & 58.33 \\
\hline $\begin{array}{l}\text { The required amount of fluid intake an adult patient per } \\
\text { day (on average) }\end{array}$ & 35 & 58.33 & 25 & 41.67 \\
\hline $\begin{array}{l}\text { Which vital signs are a part of fluid balance assessment } \\
\text { patient }\end{array}$ & 36 & 60 & 24 & 40 \\
\hline $\begin{array}{l}\text { Clinical signs associated with hypovolemia/ } \\
\text { hypervolemia }\end{array}$ & 40 & 66.67 & 20 & 33.33 \\
\hline $\begin{array}{l}\text { Nurses' action for patients who has hypovolemia/ } \\
\text { hypervolemia }\end{array}$ & 35 & 58.33 & 25 & 41.67 \\
\hline Blood products included in the fluid balance & 45 & 75 & 15 & 25 \\
\hline Fluids or drugs infused continuously & 55 & 91.67 & 5 & 8.33 \\
\hline Factors affecting urine output & 25 & 41.67 & 35 & 58.33 \\
\hline
\end{tabular}

Table (II) Critical care nurses' perception regarding fluid balance monitoring in ICUs. This table shows nurses' perception toward monitoring fluid balance in ICUs. Regarding importance of fluid balance monitoring, it was noted that more than half of nurses (53.33\%) agreed that recording the intake and output is as important as other patient care activities, $52(88.33 \%)$ of nurses agreed that fluid balance assessment is important to guide nursing care in critically ill patients and most of nurses (91.67\%) agreed that inaccurate fluid balance calculation can be a risk for the critically ill patient. As for the responsible persons for fluid balance monitoring, 52 $(88.33 \%)$ of nurses disagreed that too many people fill in one patient's fluid balance chart while $50(83.34 \%)$ of nurses agreed that the nurse is the only person responsible for a correct fluid balance calculation.

As regards fluid balance monitoring sheet, more than two thirds of nurses $42(70 \%)$ were satisfied with it while nearly half of nurses $27(45 \%)$ disagreed that the space to write the fluid numbers on the chart is adequate and three quarters of nurses disagreed that fluid balance information is recorded in too many different places on critical care observation \& patient records. In addition, two thirds of nurses $(66.67 \%)$ agreed that nurses may be responsible for more than one patient, so it is difficult to supervise all the fluid balance activities and $52(88.33 \%)$ of nurses agreed that the 24 -hour fluid balance is correctly calculated all the time.

Table (II) Critical care nurses' perception regarding fluid balance monitoring in ICUs (Total number $=60$ )

\begin{tabular}{|c|c|c|c|c|c|c|c|c|c|c|}
\hline \multirow[t]{2}{*}{ Items of nurses' perception } & \multicolumn{2}{|c|}{$\begin{array}{l}\text { Strongly } \\
\text { Disagree }\end{array}$} & \multicolumn{2}{|c|}{ Disagree } & \multicolumn{2}{|c|}{ Uncertain } & \multicolumn{2}{|c|}{ Agree } & \multicolumn{2}{|c|}{$\begin{array}{l}\text { Strongly } \\
\text { Agree }\end{array}$} \\
\hline & $\mathbf{N}$ & $\%$ & $\mathbf{N}$ & $\%$ & $\mathbf{N}$ & $\%$ & $\mathbf{N}$ & $\%$ & $\mathbf{N}$ & $\%$ \\
\hline \multicolumn{11}{|c|}{ Importance of fluid balance monitoring } \\
\hline Recording the intake and output is important as other patient care activities & 6 & 10 & 17 & 28.33 & 5 & 8.34 & 15 & 25 & 17 & 28.33 \\
\hline $\begin{array}{l}\text { Fluid balance assessment is important to guide nursing care in critically ill } \\
\text { patients }\end{array}$ & $\mathbf{0}$ & $\mathbf{0}$ & 7 & 11.67 & $\mathbf{0}$ & $\mathbf{0}$ & 20 & 33.33 & 33 & 55 \\
\hline Inaccurate fluid balance calculation can be a risk for the critically ill patient & $\mathbf{0}$ & $\mathbf{0}$ & $\mathbf{0}$ & $\mathbf{0}$ & 5 & 8.34 & 12 & 20 & 43 & 71.67 \\
\hline \multicolumn{11}{|l|}{ Responsible persons for fluid balance monitoring } \\
\hline The nurse is the only person responsible for a correct fluid balance calculation & $\mathbf{0}$ & $\mathbf{0}$ & 5 & 8.34 & 5 & 8.34 & 10 & 16.67 & 40 & 66.67 \\
\hline Too many people fill in one patient's fluid balance chart. & 0 & $\mathbf{0}$ & 27 & 45 & 25 & 41.67 & 8 & 13.33 & $\mathbf{0}$ & $\mathbf{0}$ \\
\hline \multicolumn{11}{|c|}{ Fluid balance monitoring sheet } \\
\hline Nurses are satisfied with the design of the fluid balance chart sheet & $\mathbf{0}$ & 0 & $\mathbf{0}$ & $\mathbf{0}$ & 18 & 30 & 22 & 36.67 & 20 & 33.33 \\
\hline The space to write the fluid numbers on the chart is adequate & 10 & 16.67 & 17 & 28.33 & 5 & 8.33 & 5 & 8.34 & 23 & 38.33 \\
\hline $\begin{array}{l}\text { Fluid balance information is recorded in too many different places on critical } \\
\text { care observation \& patient records }\end{array}$ & 40 & 66.66 & 5 & 8.34 & 5 & 8.33 & 10 & 16.67 & $\mathbf{0}$ & 0 \\
\hline \multicolumn{11}{|c|}{ Fluid balance calculation } \\
\hline $\begin{array}{l}\text { Nurses may be responsible for more than one patient, so it is difficult to } \\
\text { supervise all the fluid balance activities. }\end{array}$ & 13 & 21.67 & $\mathbf{0}$ & 0 & 7 & 11.67 & 10 & 16.67 & 30 & 50 \\
\hline The 24-hour fluid balance is correctly calculated all the time & $\mathbf{0}$ & 0 & 7 & 11.67 & $\mathbf{0}$ & $\mathbf{0}$ & 20 & 33.33 & 33 & 55 \\
\hline
\end{tabular}


Table (III) shows a comparison between critical care nurses' perception and knowledge regarding fluid balance monitoring in ICUs, as nurses' knowledge was moderately adequate (61.67\%), nurses' perception was favorable $(71 \%)$ and the association between perception and knowledge was considered very statistically significant $(\mathrm{p}=0.0012)$.

Table (III) Comparison between critical care nurses' perception and knowledge regarding fluid balance monitoring in ICUs.

\begin{tabular}{|l|l|l|l|l|}
\hline \multirow{2}{*}{ Items } & \multicolumn{2}{|l|}{ Total N=60 } & \multirow{2}{*}{$\mathrm{p}$} \\
\cline { 3 - 4 } & $\mathrm{N}$ & $\%$ & \\
\hline \multirow{3}{*}{ Perception } & Favorable & 426 & 71 & \multirow{3}{*}{$0.0012^{*}$} \\
\cline { 2 - 4 } & Unfavorable & 174 & 29 & \\
\hline \multirow{2}{*}{ Knowledge } & Moderately adequate & 296 & 61.67 & \\
\cline { 2 - 4 } & Inadequate & 184 & 38.33 & \\
\hline
\end{tabular}

*Significant at $5 \%$ level

Nurses reported a number of factors affecting fluid balance monitoring in ICU in table (IV) mainly inaccuracy of the measuring equipment / continuous intravenous infusions, time management (100\%), workload $(91.67 \%)$, lack of skills/ training (33.33\%), lack of knowledge, and communication with other health-care workers $(25 \%)$. Nurses' recommendations for accurate fluid balance monitoring were establishing a system for monitoring continuous infusions, which are administered through syringe pump and infusion pump as it might be duplicated or missed when monitoring fluid balance. In addition, nurses reported that they need a more accurate tool to record vomiting and hematemesis.

Table (IV) Factors affecting fluid balance monitoring as perceived by nurses in in ICUs

\begin{tabular}{|ll|l|l|}
\hline Factors & $\mathrm{N}=60$ \\
\cline { 3 - 5 } & $\mathrm{No}$ & $\%$ \\
\hline 1. & Inaccuracy of the measuring equipment / continuous intravenous infusions & 60 & 100 \\
\hline 2. & Time management & 60 & 100 \\
\hline 3. & Work load & 55 & 91.67 \\
\hline 4. & Lack of skills / training & 20 & 33.33 \\
\hline 5. & Lack of knowledge & 15 & 25 \\
\hline 6. & Communication with other health-care workers & 15 & 25 \\
\hline
\end{tabular}

\section{Discussion}

Maintaining the correct balance of fluid in the body is crucial to health ${ }^{(16)}$. However, according to Care Quality Commission (2011) ${ }^{(17)}$ recent report, enough fluids are not being given for some patients. Nurses play an important role in assessing and monitoring fluid balance ${ }^{(1-3)}$. Just $5-10 \%$ fluctuation in fluid volume can result in an adverse effect on health. Scales and Pilsworth $(2008){ }^{(9)}$ identified three main elements for fluid balance monitoring which are clinical assessment, blood chemistry and fluid balance charts.

All fluid intake and output must be documented using quantifiable amounts. Accuracy of fluid balance monitoring is a component assuring safe patient care, and is of particular importance in caring for critically ill patients. Accurate documentation is essential in fluid balance monitoring. Missing or wrongly noted numbers can influence the patient's outcome severely ${ }^{(16-17)}$. The use of fluid balance charts that show cumulative input and output is being questioned now in the literature ${ }^{(11)}$. Perren et al (2011) ${ }^{(4)}$ suggested that cumulative fluid balance charts are not accurate in a large percentage of critically ill patients and their use should be examined.

Walker et al (2012) ${ }^{(18)}$ examined 53 intravenous fluid (IV) prescription charts before IV administration and 48 after administration, the researchers noticed that accurate completion of fluid balance charts by nursing staff led to a greater assessment and documentation of fluid balance status by medical staff compared to patients where the charts had not been completed. In this regards, Vincent et al (2015) ${ }^{(19)}$ performed an audit on fluid balance monitoring for 117 patients and found showed that less than half of fluid balance charts were fully completed.

On assessing the accuracy of monitoring fluid balance in the current study, it was found that around two thirds of the recorded fluid balance was accurate and the inaccurate fluid balance recorded was fluctuating between positive and negative balance. The results of the current study are in agreement with the results of Perren et al ${ }^{(4)}$ who found that fluid balance was inaccurate in 49 of $174(33 \%)$ critically ill patients with errors ranging from $-3606 \mathrm{~mL}$ to $+2020 \mathrm{~mL}$. In addition, Diacon and Bell $(2014)^{(10)}$ investigated the recording and accuracy of fluid balance monitoring in critically ill patients and found that $79 \%$ of the original recorded fluid balance deviated by more than $50 \mathrm{~mL}$ from the required balance.

Johnson and Monckhouse (2009) ${ }^{(20)}$ found a discrepancy between fluids ordered and fluids recorded as administered. In addition, Perren et $\mathrm{al}^{(4)}$ concluded that the fluid balance charting practice is not predictive for actual changes in critically ill patients' weight and by consequence inadequately reflects fluctuations in total

\begin{tabular}{llr|}
\hline DOI: $10.9790 / 1959-0504015362$ & www.iosrjournals.org & 59|Page
\end{tabular}


body water. The results of the current study may be attributed to administration of continuous intravenous infusions and /or diuretics, which may be overlapped or missed in recording 24-h fluid balance record. Error in calculation may be another reason and the researcher in some fluid balance records noted it.

As for critical care nurses' knowledge regarding fluid balance monitoring in ICUs in the current study, it was found that the level of nurses' knowledge regarding fluid balance monitoring in ICUs was moderately adequate for around two thirds of nurses while more than a third of nurses their knowledge were inadequate. Vijayan (2011) ${ }^{(14)}$ conducted a study to assess the practices and knowledge of nurses regarding fluid and electrolyte administration in post-operative cardiac surgical patients admitted in ICU. Vijayan found that half of nurses have adequate knowledge, $47.5 \%$ of nurses have moderately adequate knowledge and the rest of nurses have inadequate knowledge ${ }^{(14)}$. In addition, Scales and Pilsworth ${ }^{(9)}$ stated that nurses' knowledge regarding fluid balance should be improved because nurses are the primary persons responsible for the monitoring of the fluid balance.

Mogileeswari and Ruth (2016) ${ }^{(21)}$ conducted a study to assess nurses' knowledge and practice regarding fluid therapy. The researchers found that $15 \%$ of nurses have adequate knowledge, $62 \%$ have moderately adequate knowledge and $23 \%$ have inadequate knowledge. While in the same study, $37 \%$ of nurses had safe practice regarding fluid therapy, $42 \%$ had moderately safe practice and $21 \%$ of nurses their practice was considered unsafe. However, Diacon and Bell $(2014)^{(15)}$ reported that critical care nurses are equipped with theoretical and practical knowledge about fluid balance monitoring.

Vincent and Mahendiran (2015) ${ }^{(22)}$ used e-Learning and verbal presentation in their study to raise nurses' awareness around fluid balance, after intervention, the researchers found a 93\% reduction in unnecessary monitoring, with corresponding increases in completion (40\%) and accuracy (48\%) of remaining charts. To improve nursing practice, adequate knowledge is needed. The results of the current study may be because fluid balance monitoring is considered a routine nursing measure; nurses may think that they are competent in fluid balance calculation and monitoring and do not look forward updating their knowledge.

Critical care nurses' perception toward monitoring fluid balance in critically ill patients. The importance of fluid balance monitoring has been shown in several studies with regard to the outcome of the critically ill patient ${ }^{(2-8)}$. In the current study, nurses agreed that maintaining fluid balance is important for critically ill patients. The result is similar to Diacon s' results as nurses reported that recording the fluid balance plays an important role in their nursing care ${ }^{(10)}$. Culleiton and Simko $(2011)^{(13)}$ stated that critical care nurses should be able to diagnose and respond to fluid balance irregularities. In the same line, Elliot et al. ${ }^{(12)}$ have pointed out that fluid balance plays an essential role in nursing management, as preserving homeostasis is crucial to maintaining optimal tissue perfusion.

On the other hand, only fourteen percent of nurses did not think that fluid balance was important ${ }^{(13)}$. However, Vincent et al $(2015)^{(19)}$ conducted a survey of nurses and to assess their views about fluid balance monitoring. In this survey, $72 \%$ of nurses said that input/output charts were being started unnecessarily in too many patients and $91 \%$ of nurses that monitoring was being continued for longer than necessary during inpatient stay.

As for the responsible persons for fluid balance monitoring. Nurses disagreed that too many people fill in one patient's fluid balance chart and agreed that the nurse is the only person responsible for a correct fluid balance calculation. However some nurses in another study, agreed with that many people fill in one patient's fluid balance chart ${ }^{(15)}$. In Reid et al. study ${ }^{(23)}$, nobody felt responsible for the accurate documentation and suggested that the allocated person should sign for the accurate recording of the data.

As for fluid, balance monitoring sheet. Nurses in the current study, agreed that the fluid balance chart was well designed in their units. While nearly half of nurses agreed that, the space to write the fluid numbers on the chart is inadequate. In other study, a quarter of nurses felt the space was too small, while seventy-four percent of the nurses expressed satisfaction with the space to write the numbers ${ }^{(15)}$. Bennet $(2010)^{(11)}$ recommended that the fluid balance chart should be assessed and updated regularly and designed for each intensive care unit individually.

Fluid balance calculation. More than two thirds in the current study reported that it was easy to manage fluid balance activities for patients. On the contrary, twenty-six percent of nurses in this study agreed that it is difficult to supervise all the fluid balance activities because they may be responsible for more than one patient. Some nurses in a study explained this because the ICU is busy or nurses have two patients to care for them ${ }^{(15)}$. The main cause of inaccurate fluid balance monitoring was a shortage of nursing staff as indicated in another study ${ }^{(18)}$. Nurses' shortage and workload in ICU may be reasons for inaccurate fluid balance monitoring in the current study. 
In addition, the majority of nurses in the current study reported that the 24-hour fluid balance is correctly calculated all the time. Similarly, nurses in many studies reported the same results, as they believed that fluid balance activities are manageable ${ }^{(10,14,15,18,19)}$. However, there is a gap between nurses' perception and actual situations because inaccurate fluid balance was found in $35 \%$ of fluid balance in the current study. In the current study, the overall nurses' perception was favorable and the association between perception and knowledge was considered very statistically significant. This can be explained as if nurses have adequate knowledge their perception will be favorable

Inaccuracy of the measuring equipment / continuous intravenous infusions, time management, workload, lack of skills/ training, lack of knowledge, and communication with other health-care workers are factors affecting fluid balance monitoring in ICU as reported by nurses in the current study. Nurses' recommendations for accurate fluid balance monitoring were establishing a system for monitoring continuous infusions, which are administered through syringe pump and infusion pump as it might be duplicated or missed when monitoring fluid balance. In addition, nurses reported that they need a more accurate tool to record vomiting and hematemesis.

The results of this study are similar to Reid et al. ${ }^{(23)}$ study results as they found that a shortage of nursing staff, a deficit in knowledge and a heavy workload are factors affecting fluid balance monitoring in ICU. Scribante and Bhagwanjee (2007) ${ }^{(24)}$ showed that shortage of nurses and workload are important issues in nursing. In addition, Scales and Pilsworth ${ }^{(9)}$ emphasized the importance of improving nurses' knowledge in enhancement of fluid balance monitoring practice. Herbert and Elsayed ${ }^{(8)}$ recommended that fluid prescribing guidelines should be introduced into the ICU.

Similarly, Diacon and Bell ${ }^{(15)}$ stated that the most important aspects affecting nurses' accuracy of fluid balance monitoring, recording and calculation are workload and time management, lack of training, inexperience, inaccuracy of the measuring equipment, communication with other health-care workers, distractions and uninformed visitors. The results of the current study may be attributed to lack of in-service education and training regarding the basic nursing care because nurses give more importance to advanced nursing care. In addition, shortage of nurses increase workload on the existing staff may lead to errors in monitoring fluid balance.

In Vincent et al ${ }^{(19)}$ project nurses suggested that if they have more guidance from the medical team regarding monitoring and more education on fluid balance, it would be helpful. In Diacon and Bell study ${ }^{(15)}$, the solutions to improve fluid balance monitoring are training of all staff, clear standardized charts, clear prescriptions from doctor, calculator at bedside, documentation audits, information signs at the bedside, basic calculation training and infusion pumps. In the current study nurses suggestion for accurate fluid balance monitoring were establishing a system for monitoring continuous infusions and a more accurate tool to record vomiting and hematemesis. Nurses' suggestions reflect their actual needs as they face a problem in monitoring continuous infusions.

\section{Conclusion}

Two thirds of the recorded fluid balance was accurate and the inaccurate fluid balance recorded was fluctuating between positive and negative balance. The level of nurses' knowledge regarding fluid balance monitoring in ICUs was moderately adequate. Nurses had favorable perception regarding fluid balance monitoring. There was a significant association between nurses' knowledge and perception regarding fluid balance monitoring. Nurses reported that inaccuracy of the measuring equipment / continuous intravenous infusions, time management, workload, lack of skills/ training, lack of knowledge, and communication with other health-care workers are factors affecting fluid balance monitoring in ICU.

\section{Recommendations}

Fluid balance monitoring is a basic nursing intervention. Hospital and ICU administrators pay more attention for advanced and sophisticated care than basic nursing care therefore; basic nursing intervention is treated as a routine and not as important as other nursing intervention. Therefore, Hospital and ICU administrators should pay more attention for basic nursing interventions especially monitoring of fluid balance in ICU. For accuracy of fluid balance monitoring in ICU, awareness around the potential consequences of calculation errors should be reinforced during patient discussions and in-service education should be conducted to improve nurses' knowledge. Novice nurses should be trained adequately regarding assessment and monitoring of fluid balance. The requirement to provide accurate, correct fluid balance monitoring and recording as part of the patient's vital sign data should be established as a fundamental standard of practice for every nurse practicing in an ICU. Fluid balance chart should be updated and a system for monitoring continuous infusions and a more accurate tool to record vomiting and hematemesis should be established. Further studies may assist in refining the particular challenges of accurate fluid balance monitoring. 
Limitations of the study; the small size of the sample and the use of only one hospital group could have limited the generalization of the study results. Body weight, nutritional needs, sensible and insensible losses were not estimated in this study. A qualitative research approach in the form of interviews could give more profound perspectives of the nurses with regard to fluid balance monitoring.

\section{References}

[1]. Gonzalez F, Vincent F. The fluid balance in the critically ill patients: what are we talking about? Minerva Anestesiol 2011;77(8):766-7.

[2]. Ind D. Fluid Assessment. Renal Society of Australasia Journal2006; 2 (3):51-2

[3]. Shepherd A. Measuring and managing fluid balance. Nursing Times2011;107(28):12-6.

[4]. Perren A, Markmann M, Merlani G, Marone C, Merlani P. Fluid balance in critically ill patients Should we really rely on it? Minerva Anestesiol 2011;77(8):802-11.

[5]. Bouchard J, Soroko S, Chertow G, Himmelfarb J, Ikizler T, Paganini E et al. Fluid accumulation, survival and recovery of kidney function in critically ill patients with acute kidney injury. Kidney Int 2009;76:422-7.

[6. Boyd J, Forbes J, Nakada T, Walley K, Russell J. Fluid resuscitation in septic shock: A positive fluid balance and elevated central venous pressure are associated with increased mortality. Crit Care Med 2011;39(2):259-65.

[7]. Payen D, de Pont A, Sakr Y, Spies C, Reinhart K, Vincent JL. A positive fluid balance is associated with a worse outcome in patients with acute renal failure. Crit Care 2008; 12(3):R74. http://ccforum.com/content/12/3/R74 (retrieved on October 27, 2014).

[8]. Herbert L, Elsayed M. Fluid and Electrolyte Balance in the Intensive Care Unit - Trickle or Flood ?http:// www. appraisal. nes.scot.nhs.uk/media/179709/fluid-poster.pdf retrieved May 14, 2016.

[9]. Scales K, Pilsworth J. The importance of fluid balance in clinical practice. Nursing Standard 2008; 22: 47, 50-7.

[10]. Diacon A, Bell J. Investigating the recording and accuracy of fluid balance moni toring in critically ill patients. S Afr J Crit Care 2014;30(2):55- 7 .

[11]. Bennett C. 'At A Glance' Fluid Balance Bar Chart. London: NHS Institute for Innovation and Improvement 2010. tinyurl.com/fluid-balance. Retrieved on June 15, 2015.

[12]. Elliot D, Aitken L, Chaboyer W. ACCCN's Critical Care Nursing, 1st ed. Marrickville, Australia: Mosby Elsevier,2007:440-6

[13]. Culleiton AL, Simko LC. Keeping electrolytes and fluids in balance. Nursing2013 Critical Care 2011;6(2):30-5.

[14]. Vijayan A. A study to assess the knowledge and practices of staff nurses regarding fluid and electrolyte administration in postoperative cardiac surgical patients admitted in cardiac surgical ICU and cardiac surgical ward. Sree Chitra Tirunal Institute. In press 2011.

[15]. Diacon A, Bell J. Fluid balance monitoring in critically ill patients. Faculty of Medicine and Health Sciences at Stellenbosch University. In press 2012.

[16]. Welch K. Fluid balance. Learning Disability Practice2010; 13: 6, 33-8.

[17]. Care Quality Commission: Dignity and Nutrition for Older People: Review of Compliance. London: CQC2011. tinyurl.com/QCQworcestershire

[18]. Walker G, Stewart-Parker E, Chinthapalli S, Ostermann M, Dargan P, Wood D. Intravenous fluid use in the acutely unwell adult medical inpatient: improving practice through a clinical audit process. J R Coll Physicians Edinb 2012; 42:211-5.

[19]. Vincent M, Mahendiran T, Nava S, Winterbottom J, Myers J, Perera R, Srikumaran S. Fluid balance monitoring: a Quality Improvement project. BMJ Qual Improv Report 2015;4.

[20]. Johnson R, Monkhouse S. Postoperative fluid and electrolyte balance: alarming audit results. Journal of Perioperative Practice2009, 19(9):291-4.

[21]. Mogileeswari P, Ruth M. Knowledge and practice regarding fluid and electrolyte replacement therapy for patient with burns. International Journal of Multidisciplinary Research and Development 2016;3(4):217-20.

[22]. Vincent M, Mahendiran T. Improvement of fluid balance monitoring through education and rationalization. BMJ Quality Improvement Reports 2015; u209885.w4087 doi: 10.1136/bmjquality.u209885.w4087. http://qir.bmj.com/ retrieved on May 14, 2016.

[23]. Reid J. Improving the monitoring and assessment of fluid balance. Nursing Times2004; 100:20, 36-9.

[24]. Scribante J, Bhagwanjee S. National audit of critical care resources in South Africa - nursing profile. S Afr Med J. 2007;97(12):1315-8. 